\title{
Bright, high-repetition-rate water window soft $X$-ray source enabled by nonlinear pulse self-compression in an antiresonant hollow-core fibre
}

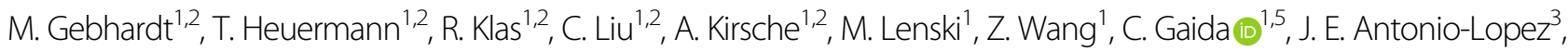 \\ A. Schülzgen ${ }^{3}$, R. Amezcua-Correa ${ }^{3}$, J. Rothhardt ${ }^{1,2,4}$ and J. Limpert ${ }^{1,2,4}$
}

\begin{abstract}
Bright, coherent soft X-ray radiation is essential to a variety of applications in fundamental research and life sciences. To date, a high photon flux in this spectral region can only be delivered by synchrotrons, free-electron lasers or highorder harmonic generation sources, which are driven by kHz-class repetition rate lasers with very high peak powers. Here, we establish a novel route toward powerful and easy-to-use SXR sources by presenting a compact experiment in which nonlinear pulse self-compression to the few-cycle regime is combined with phase-matched high-order harmonic generation in a single, helium-filled antiresonant hollow-core fibre. This enables the first $100 \mathrm{kHz}$-class repetition rate, table-top soft $\mathrm{X}$-ray source that delivers an application-relevant flux of $2.8 \times 10^{6}$ photon s $\mathrm{eV}^{-1}$ around $300 \mathrm{eV}$. The fibre integration of temporal pulse self-compression (leading to the formation of the necessary strong-field waveforms) and pressure-controlled phase matching will allow compact, high-repetition-rate laser technology, including commercially available systems, to drive simple and cost-effective, coherent high-flux soft X-ray sources.
\end{abstract}

Laser-driven soft X-ray (SXR) sources based on highorder harmonic generation $(\mathrm{HHG})^{1}$ are known for their table-top dimensions, excellent spatial coherence and ultrashort pulse durations ${ }^{2}$, which make them attractive tools for advanced spectroscopy ${ }^{3,4}$. Additionally, they are expected to enable area-wide evolution of lens-less imaging in the water window ${ }^{5}$, and they hold great promise for the production of isolated attosecond pulses shorter than the atomic unit of time ${ }^{6,7}$.

In the past decade, water window HHG has mostly been achieved with the help of optical parametric amplifiers operating at a wavelength of approximately $2 \mu \mathrm{m}^{4,8-11}$.

\footnotetext{
Correspondence: M. Gebhardt (martin.gebhardt@uni-jena.de) ${ }^{1}$ Institute of Applied Physics, Abbe Center of Photonics, Friedrich-SchillerUniversität Jena, Albert-Einstein-Str. 15, 07745 Jena, Germany ${ }^{2}$ Helmholtz-Institute Jena, Fröbelstieg 3, 07743 Jena, Germany Full list of author information is available at the end of the article
}

This driving wavelength is identified as a "sweet spot" for pushing the phase-matched harmonic energy $\left(\sim \lambda^{1.4-1.7}\right)^{12}$ beyond the carbon K-edge, while the single-atom response $\left(\sim \lambda^{-(5-6)}\right)^{13}$ is still reasonable and can partially be compensated for by high phase matching pressures ${ }^{14}$. To date, the reported generated photon flux around $300 \mathrm{eV}$ is as high as $1 \times 10^{9}$ photons $\mathrm{s}^{-1} \mathrm{eV}^{-1}$ at a $1 \mathrm{kHz}$ repetition rate based on HHG in a gas-filled capillary ${ }^{4}$. To achieve high flux levels, the typical experimental conditions require $>40 \mathrm{GW}$ peak power (Supplement), which implies the generation and handling of multi-mJ energy ${ }^{4,9}$, or few-cycle pulses ${ }^{11,15}$. These experimental constraints alter the fundamentally desired user-friendly and straightforward nature of laser-based SXR sources and are responsible for the fact that subsequent work on applications is often closely related to source development ${ }^{4,11}$. Additionally, techniques such as coincidence detection ${ }^{16}$

\section{(c) The Author(s) 2021}

(c) (i) Open Access This article is licensed under a Creative Commons Attribution 4.0 International License, which permits use, sharing, adaptation, distribution and reproduction in any medium or format, as long as you give appropriate credit to the original author(s) and the source, provide a link to the Creative Commons license, and indicate if changes were made. The images or other third party material in this article are included in the article's Creative Commons license, unless indicated otherwise in a credit line to the material. If material is not included in the article's Creative Commons license and your intended use is not permitted by statutory regulation or exceeds the permitted use, you will need to obtain permission directly from the copyright holder. To view a copy of this license, visit http://creativecommons.org/licenses/by/4.0/. 


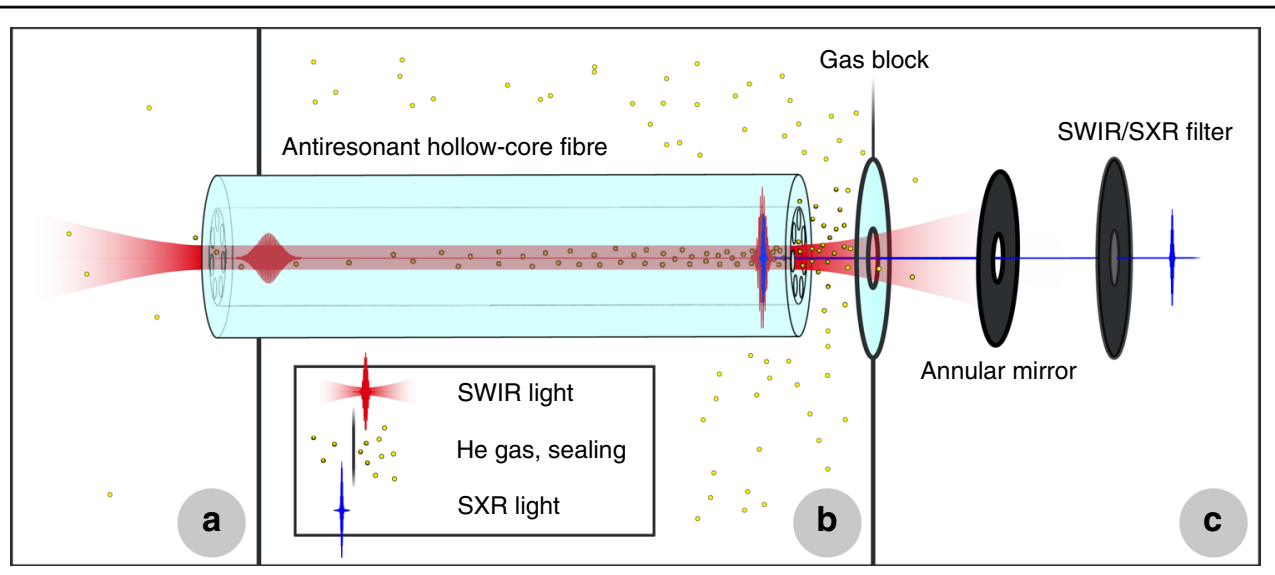

Fig. 1 Nonlinear pulse self-compression and HHG setup. a Input coupling within helium gas at atmospheric pressure. $\mathbf{b}$ The ARHCF (core diameter: $84.5 \mu \mathrm{m}$, length: $120 \mathrm{~cm}$ ) is differentially pumped with the output facet located in a high-pressure chamber. Near the fibre output, the highpressure region is separated from the following vacuum chamber by a $1 \mathrm{~mm}$ thick sapphire window that has a conical hole with diameters of 100-250 $\mathrm{mm}$. This gas block prevents most of the helium from reaching the following low-pressure region, while the light emerging from the fibre passes through it. To achieve this, the ARHCF output (located approximately $2 \mathrm{~mm}$ from the gas block) is movable in the horizontal and vertical directions (only the optimal positioning is shown for clarity). c The final vacuum chamber contains an annular mirror, a thin metal filter to separate the SWIR light from the generated high-order harmonics, and a flat-field imaging grating spectrometer (not shown)

and space-charge-reduced photoelectron spectroscopy ${ }^{17}$ require repetition rates $>1 \mathrm{kHz}$, where the generation and handling of high-peak-power pulses become increasingly challenging, and the reported $300 \mathrm{eV}$ flux is only approximately $4 \times 10^{4}$ photons $\mathrm{s}^{-1} \mathrm{eV}^{-1}$, based on HHG in a free-space gas target ${ }^{9,15}$. It is therefore highly desired to enable compact, high-repetition-rate laser sources (including turnkey, commercial systems) to directly generate high-flux SXR high-order harmonics.

In this work, we demonstrate an approach to SXR HHG, which is based on power-scalable laser emission from a $98 \mathrm{kHz}$ repetition rate thulium-doped fibre laser. It combines nonlinear self-compression of the driving pulses ${ }^{18}$ with waveguide $\mathrm{HHG}^{19}$ within a single, gas-filled antiresonant hollow-core fibre (ARHCF). In contrast to previous demonstrations ${ }^{4,8-11,15}$, the fibre-integrated HHG experiments require only GW-level peak power, multicycle laser pulses. This is a result of the carefully controlled intensity enhancement during pulse compression. While so-called all-fibre, table-top SXR sources, driven by mid-infrared lasers, have been briefly envisaged theoretically $^{20}$, this is the first time that this scheme is experimentally realised and analysed in depth. This is fundamentally enabled by advances in the development of $\mathrm{ARHCFs}^{21}$, which have already enabled HHG in the extreme ultraviolet ${ }^{22}$, and represents a significant step in increasing the availability and performance of highrepetition-rate sources for SXR or attosecond science.

Our experiments are enabled by a thulium-doped fibre chirped-pulse amplification system that provides $100 \mathrm{fs}$ pulses with energies up to $450 \mu \mathrm{J}$ at a central wavelength of $1910 \mathrm{~nm}$ (Methods, Supplement). To prevent absorption from water vapour or thermal failure of the fibre $\operatorname{tip}^{23}$, mode coupling to the ARHCF is performed within helium gas at 1 bar pressure (Fig. 1a). Through its output end, the fibre can be filled with helium at a pressure of up to 20 bar. Even at this pressure, the zero-dispersion wavelength is below the central wavelength of the laser pulses (Methods). Hence, these pulses self-compress as they undergo self-phase modulation while propagating to the output of the waveguide. For appropriately chosen input pulse energy and output pressure, the enhanced electric field strength close to the fibre end is high enough to directly drive HHG within the ARHCF (Fig. 1b). We note that the typical phase-matched photon energy cut-off achieved with short-wavelength infrared (SWIR) driving wavelengths is in the SXR regime ${ }^{12}$. Because of the good transparency of helium in this spectral region, the highphoton-energy portion of the generated harmonics is well transmitted to the characterisation (Fig. 1c) or subsequent experiments.

A numerical analysis of the pulse self-compression (Methods) and phase matching to the SXR light is presented in Fig. 2. The input pulse characteristics are chosen to represent the experimental conditions (Supplement). The steady-state gas particle density within the ARHCF (Fig. 2a) is retrieved from gas flow modelling (Methods), with a pressure of $3.345 \mathrm{bar}$ at the fibre output. Figure $2 \mathrm{~b}$ shows the evolution of the time-dependent on-axis intensity inside the fibre for a launched pulse energy of $265 \mu \mathrm{J}$. The nonlinear dynamics cause the pulses to selfcompress from an initial FWHM duration of $100 \mathrm{fs}$ to below $20 \mathrm{fs}$ ( 3.5 optical cycles) as they reach a position just before the fibre output. At this point, the peak intensity is 


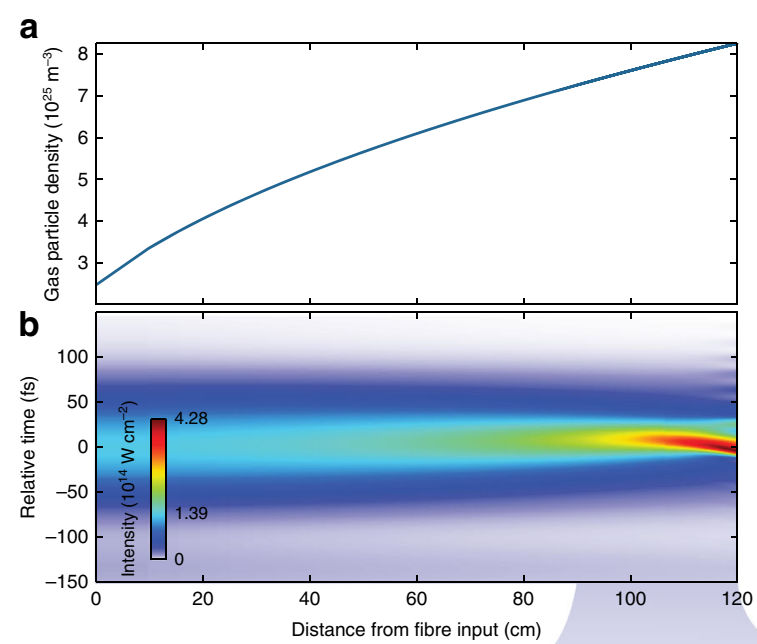

C

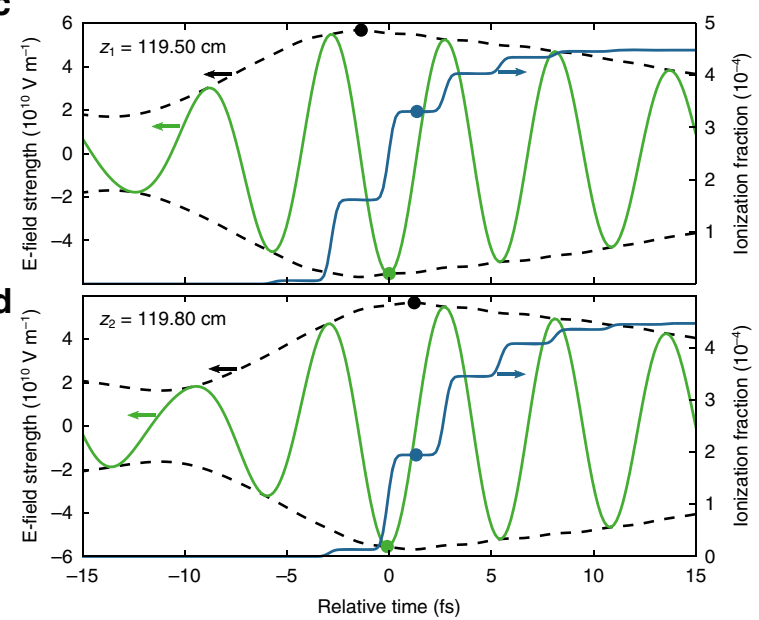

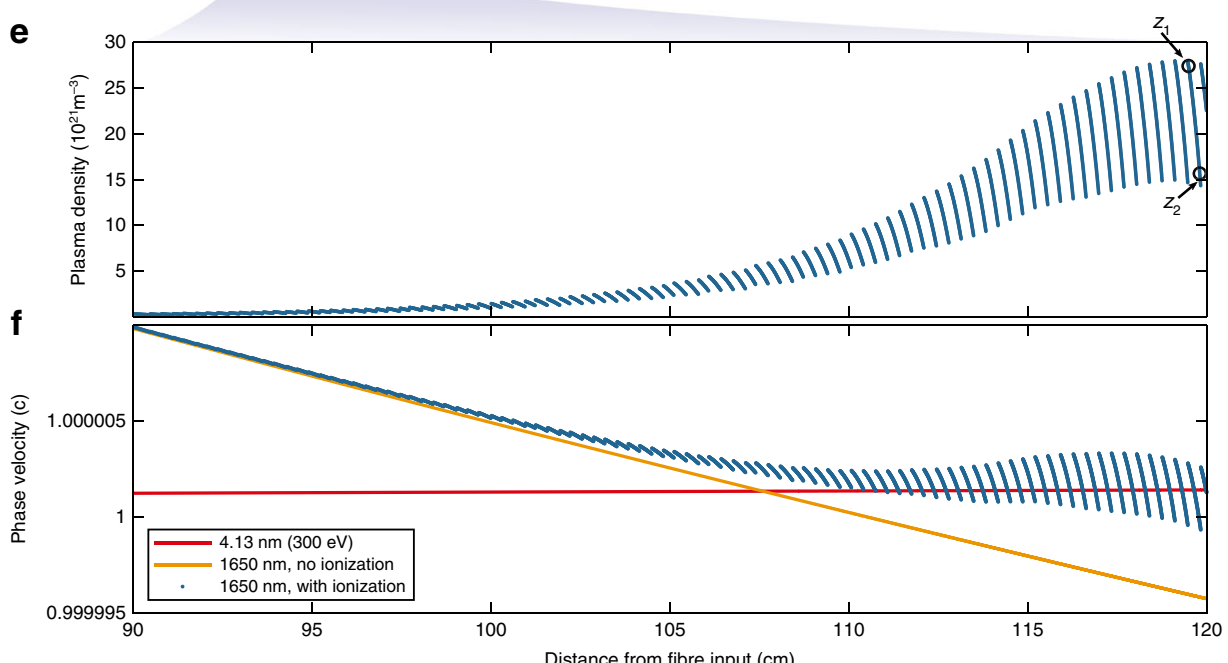

Fig. 2 Nonlinear pulse self-compression and simultaneous phase matching of SWIR and SXR light. a Helium gas particle density along the fibre for pressures of 1 bar at the input and 3.345 bar at the output. $\mathbf{b}$ Evolution of the simulated time-dependent on-axis intensity within the fibre. The reference frame velocity is the group velocity at $1910 \mathrm{~nm}$. c Simulated time-dependent electric field strength (green line), envelope (black dashed line) and ionisation fraction (blue line) at a distance $z_{1}=119.50 \mathrm{~cm}$ from the fibre input. Solid points highlight the field peak values/positions and the ionisation fraction after the global peak of the electric field. The relative time axis is centred at the electric field maximum at $z_{1}$. $\mathbf{d}$ same as (c) but at $z_{2}=119.80 \mathrm{~cm}$ from the fibre input. The reference frame velocity is the phase velocity at $1650 \mathrm{~nm}$. e Simulated on-axis plasma density after the global peak of the electric field. $\mathbf{f}$ Comparison of the SWIR and SXR phase velocities vs. distance from the fibre input

increased to $4.28 \times 10^{14} \mathrm{~W} \mathrm{~cm}^{-2}$, which causes ionisation of the gas, as seen from the acceleration of the pulse (Fig. 2b) ${ }^{24}$. To observe a significant growth of harmonic radiation, it is necessary to fulfil the phase matching conditions ${ }^{1}$ for at least one half-cycle within the SWIR pulse. Due to the significant field strength dependence of the atomic response ${ }^{25}$, phase matching close to the peak of the driving pulse is preferred. This is also favourable for macroscopic harmonic signal growth from few-cycle driving pulses because it reduces the variation in the intrinsic HHG phase with propagation distance ${ }^{14}$. Consequently, we evaluate the ionisation levels directly after the peak of the pulse. For example, Fig. 2c shows the simulated time-dependent electric field and ionisation fraction at a distance $z_{1}=119.50 \mathrm{~cm}$. This is compared to the situation at $z_{2}=119.80 \mathrm{~cm}$ (Fig. 2d). It becomes apparent that the evolution of the on-axis plasma density directly after the global electric field maximum (Fig. 2e) exhibits a modulation due to the temporal walk-off between the carrier and its envelope. This walk-off is a direct consequence of the waveguide dispersion, similar to the Gouy phase shift in a tight focusing geometry. It can be furthermore derived from the self-compressed electric fields that the instantaneous wavelength of the most intense cycle is approximately $1650 \mathrm{~nm}$. This is not surprising, as ionisation causes a blueshift of the spectrum ${ }^{24}$. 


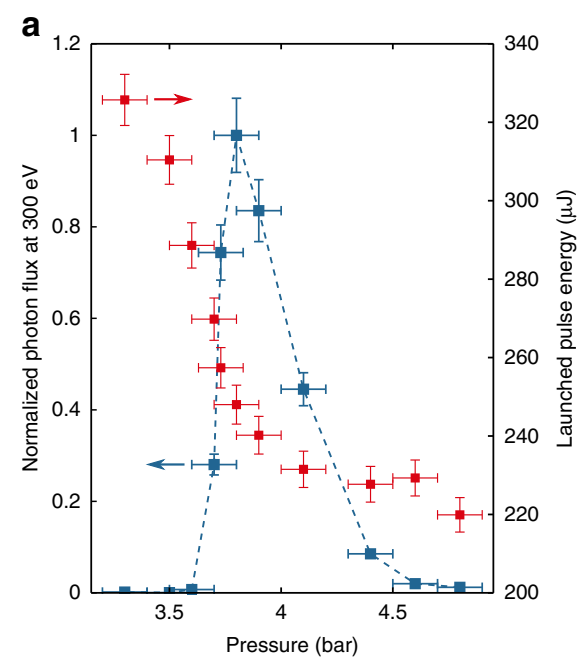

b

Fig. 3 High-harmonic generation experimental results. a Normalised generated photon flux, evaluated at $300 \mathrm{eV}$ for different helium gas pressures applied to the high-pressure chamber. The individual error bars in the $y$-direction are based on the standard deviation of 240 consecutive measurements (Supplement) and the uncertainty of the pressure reading ( 0.1 bar). Measured flux data points are connected by the blue dashed line. The right $y$-axis refers to the launched pulse energy necessary for obtaining the optimum flux at each pressure setting. b Measured HHG spectrum and divergence under optimised experimental conditions. The clipping in the divergence direction is due to the limited apertures of the SWIR/SXR separation elements. $\mathbf{c}$ Evaluation of the generated photon flux not accounting for carbon contaminants, as can be seen from the K-edge absorption feature at a $284 \mathrm{eV}$ photon energy

Finally, the above-derived quantities are used to calculate the phase velocities for the centre of the HHG driving pulse and a desired high-order harmonic wavelength. It can be seen in Fig. $2 \mathrm{f}$ that the chosen output pressure not only is crucial for the intensity enhancement achieved through pulse self-compression but also, most importantly, counteracts the ionisation-induced acceleration of the phase velocity of the driving field such that it can travel in-phase with the generated SXR light. While this situation is globally maintained over several centimetres, we find that the above-mentioned walk-off effects limit the coherent growth of the harmonic signal from an individual field half-cycle to a few millimetres (Supplement). This is consistent with the steep slope of the individual half-cycle phase velocities (Fig. 2f, blue data points) and requires fine tuning of the experimental conditions for maximum photon flux.

The experimental conditions are optimised with the goal of generating a high photon flux around $300 \mathrm{eV}$. This is done by scanning the gas pressure applied to the highpressure chamber and subsequently adjusting the launched pulse energy for optimum flux (Fig. 3a). The optimisation parameter exhibits a sharp peak around a pressure of $3.8 \mathrm{bar}$, which agrees reasonably well with the expectations from our numerical analysis (Fig. 2). A drop in flux to $\sim 1 / \mathrm{e}^{2}$ times the optimum value is observed for a relative pressure change of $10 \%$. In contrast, the pulse propagation simulations show that the overall nonlinearity of the pulse self-compression and, consequently, the peak electric field strength at the fibre output are easily restored when adjusting the launched pulse energy to a $10 \%$ pressure change. Therefore, we conclude that controlling the gas pressure allows control of the phase matching and favouring of the coherent growth of SXR light in the ARHCF. Figure 3b presents a measured HHG spectrum including the beam divergence, which is represented on the vertical axis. For the signal-optimised experimental conditions, a full-angle divergence of approximately $2 \mathrm{mrad}$ (Methods) and a phase-matched photon energy cut-off around $330 \mathrm{eV}$ are observed. In fact, the simulated, phase-matched half-cycle cut-off is $334 \mathrm{eV}$ (Supplement). In the experiment presented herein, an overall flux of $(2.8 \pm 1.8) \times 10^{6}$ photons $\mathrm{s}^{-1} \mathrm{eV}^{-1}$ is generated at $300 \mathrm{eV}$ (Fig. 3c, Methods). Using only one annular mirror and one $200 \mathrm{~nm}$ thin aluminium filter, the SWIR suppression is $>10^{6}$, with $(1.3 \pm 0.8) \times 10^{5}$ photons $\mathrm{s}^{-1} \mathrm{eV}^{-1}$ available for experiments. Subsequently, we demonstrate X-ray absorption fine-structure spectroscopy near the carbon K-edge, and we measure an integrated power RMS stability of $<5 \%$ in the water window over 20 min (Supplement). This shows that our table-top, $100 \mathrm{kHz}$-class repetition rate water window source operates at an application-relevant flux and performance level.

In conclusion, we have demonstrated nonlinear pulse self-compression and SXR HHG at a $98 \mathrm{kHz}$ repetition rate in a single ARHCF. This results in $>10^{6}$ photons $\mathrm{s}^{-1}$ $\mathrm{eV}^{-1}$ at $300 \mathrm{eV}$, directly emitted from the fibre output. This is the first time that such an experiment is reported, 
and the results show that high-repetition-rate ultrafast lasers that deliver moderate pulse peak powers can be used in a simple, integrated scheme to generate highorder harmonics up to the water window. Because the SXR yield is not absorption-limited and the involved ionisation levels are below the critical limit for phase matching, we see significant prospects for advancing the approach described herein in terms of photon flux (Supplement). Furthermore, with an increase in the driving laser wavelength ${ }^{26}$, self-compression in ARHCFs will allow direct generation of $\mathrm{keV}$ photon energies and extremely short attosecond pulses at $a \geq 100 \mathrm{kHz}$ repetition rate.

While our work opens up possibilities to study complementary techniques such as quasi-phase matching ${ }^{22}$ or driving pulse synthesis ${ }^{27}$ within the waveguide, we believe that its results are most interesting for a variety of applications that significantly benefit from compact and easy-to-use high-repetition-rate SXR sources, e.g. coincidence spectroscopy ${ }^{16}$ or table-top lens-less imaging of organic samples ${ }^{5}$. Because of the versatility of the pulse self-compression, this approach can be scaled to much higher peak powers ${ }^{18}$. However, we believe that this work represents, first and foremost, a milestone in the development of industrial-grade, laser-driven SXR sources. These sources could use ARHCFs for beam delivery ${ }^{28}$, self-compression and HHG in a single apparatus, making them more affordable and available to a much broader community in fundamental and applied sciences with medical applications in reach.

\section{Methods}

\section{Fibre laser setup}

The ultrafast fibre laser system is seeded by a commercial seed source and uses thulium-doped photonic crystal fibres within the first preamplification stages. The pulses are stretched to a duration of approximately $1 \mathrm{~ns}$ using a grating-based, Oeffner-type stretcher before further amplification and reduction of the oscillator repetition rate based on an acousto-optic modulator. The pulse train with a $98 \mathrm{kHz}$ repetition rate has an average power of approximately $200 \mathrm{~mW}$ prior to the main amplifier of the system, which consists of a thulium-doped large-pitch fibre with a core diameter of $80 \mu \mathrm{m}$. The main amplifier is pumped with a commercially available $793 \mathrm{~nm}$ diode laser and increases the average power to approximately $50 \mathrm{~W}$. After pulse compression in a grating-based Treacy-type compressor enclosed in a vacuum chamber, the output pulses carry up to $450 \mu \mathrm{J}$ of energy and have a duration (FWHM) of $100 \mathrm{fs}$.

\section{Dispersion of the ARHCF}

The dispersion of the ARHCF is modelled as described in ref. ${ }^{20}$. The fibre used in the experiments described herein has a resonance band up to a wavelength of $1380 \mathrm{~nm}$. This resonance is accounted for by an additional term added to the propagation constant ${ }^{29}$, which we use for the pulse propagation simulations. We find the zerodispersion wavelength $(<700 \mathrm{~nm}$ in this case) by numerically calculating the root of the group velocity dispersion.

\section{Calculation of the gas flow and gas density}

The gas flow through the ARHCF is modelled as described in $^{30}$, assuming a circular tube with a $84.5 \mu \mathrm{m}$ diameter and a $120 \mathrm{~cm}$ length. It can be shown that the continuum component of the flow strongly dominates over the molecular flow component, allowing us to use the well-known description for the pressure and density distribution $^{31}$

$$
p(z)=\sqrt{p_{0}^{2}+\frac{z}{L}\left(p_{L}^{2}-p_{0}^{2}\right)}
$$

where $p_{L}$ and $p_{0}$ are the fixed pressures at $z=L$, the fibre output, and at $z=0$, the fibre input.

\section{Simulation of pulse propagation}

Numerical simulations of pulse propagation in the fibre are performed based on the unidirectional field propagation equation for the fundamental mode $^{32}$ :

$$
\begin{aligned}
\partial_{z} E(z, \omega)= & i\left(\beta(\omega)-\frac{\omega}{v}\right) E(z, \omega) \\
& +i \frac{\omega^{2}}{2 c^{2} \varepsilon_{0} \beta(\omega)} P^{\mathrm{NL}}(z, \omega)
\end{aligned}
$$

Here, $E$ is the electric field amplitude in the spectral domain, $\omega$ is the angular frequency, $\beta$ is the fundamental mode propagation constant, $v$ is the reference frame velocity, $c$ is the vacuum speed of light, $\epsilon_{0}$ is the vacuum permittivity and $P^{\mathrm{NL}}$ is the nonlinear polarisation. $P^{\mathrm{NL}}$ is influenced by the Kerr nonlinearity and plasma formation, which we include in the simulations by calculating the Ammosov-Delone-Krainov ionisation rates ${ }^{33}$.

The fibre is designed for fundamental mode operation, which is consistent with the absence of higher order modes at the fibre output during the experiments. The plasma density in this work is still moderate, from which we deduce that coupling of the power to higher order modes during propagation is not significant. Therefore, pulse propagation is described in the fundamental mode only, for which we find good agreement with the experiments.

\section{Calculation of phase velocities}

The phase velocity of the high-order harmonics is calculated from the refractive index data available in ref. ${ }^{34}$. For the driving field propagation constant, which gives straightforward access to its phase velocity, we make the 
approximation described in $^{1}$ :

$$
\begin{aligned}
\beta(\omega)= & \frac{\omega}{c} \cdot\left(1+\frac{p}{p_{0}}(1-\eta) \cdot \delta\right) \\
& -\frac{2 \pi c}{\omega} \cdot\left(\frac{p}{p_{0}} \eta N_{\mathrm{atm}} r_{\mathrm{e}}+\frac{2.4048^{2}}{4 \pi a^{2}}\right)
\end{aligned}
$$

where $p$ and $p_{0}$ are the experimental pressure and the pressure under standard conditions, $\eta$ is the ionisation fraction, $\delta$ is the neutral gas dispersion, $N_{\mathrm{atm}}$ is the number density under standard conditions, $r_{\mathrm{e}}$ is the classical electron radius and $a$ is the core radius of the hollow fibre (see Supplement for definition).

\section{Determination of the launched pulse energy}

In low-power operation, the overall transmission of the $120 \mathrm{~cm}$ long ARHCF is approximately 90\%. During the HHG experiments, we perform relative power monitoring of the output power, which is used together with the simulation results to determine the experimental launched pulse energy, as shown in Fig. 3a. This gives 86\% transmission for the experimental conditions of the optimal HHG signal (see Table S1).

\section{Characterisation of the HHG flux}

The photon flux is estimated from the spectral characterisation of the HHG signal. This method is discussed in more detail in ref. ${ }^{35}$, where the flux in photons per second, $N_{\mathrm{ph}, \mathrm{s}}$, is given as:

$$
N_{\mathrm{ph}, \mathrm{s}}=\frac{S_{\mathrm{CCD}} \cdot \sigma}{\eta_{\mathrm{QE}} \cdot E_{\mathrm{ph}}^{[\mathrm{bg}]} \cdot \eta_{\mathrm{g}} \cdot t_{\mathrm{f}} \cdot t_{\mathrm{gas}} \cdot t_{\mathrm{exp}}}
$$

Here, $S_{\mathrm{CCD}}$ is the signal (counts) measured on the detector, $\sigma$ and $\eta_{\mathrm{QE}}$ are the CCD sensitivity and CCD quantum efficiency, and $E_{\mathrm{ph}}^{\mathrm{bg}]}$ is the evaluated HHG photon energy in units of a bandgap energy of $3.65 \mathrm{eV}$. The factor $\sigma /\left(\eta_{\mathrm{QE}} \cdot E_{\mathrm{ph}}^{[\mathrm{bg}]}\right)$ has been calibrated for the CCD in use by the Physikalisch-Technische Bundesanstalt (PTB). $\eta_{\mathrm{g}}$ is the grating diffraction efficiency (retrieved from measurements using free-electron-laser radiation $\left.{ }^{36}\right), t_{\mathrm{f}}$ is the transmission of the $200 \mathrm{~nm}$ aluminium filter used (which is measured experimentally), and $t_{\mathrm{gas}}$ is the theoretical transmission through the helium gas, consisting of the high-pressure region (see the main text) and the lowpressure spectrometer chamber, for which we determine a propagation length of $1.25 \mathrm{~m}$ and a pressure of 0.7 mbar. Finally, $t_{\exp }$ is the exposure time of the measurement. In addition to these corrections, the clipping of the HHG beam, shown in Fig. 3b, is corrected. For this purpose, a Gaussian profile is fitted to the vertical outline of the signal at $300 \mathrm{eV}$. This evaluation also gives an estimated full divergence angle of $2 \mathrm{mrad}$. The combined relative uncertainties of the quantities mentioned above give a worst-case error estimation of $\pm 64 \%$ for the absolute flux value.

The visible dip at approximately $284 \mathrm{eV}$ (Fig. 3b, c) is associated with the K-shell absorption in carbon and relates to a hydrocarbon contaminant, which is most likely deposited on the spectrometer grating. The contaminant absorption is equivalent to that of a $35 \mathrm{~nm}$ thick pure carbon layer.

\section{Acknowledgements \\ The authors would like to thank Prof. Adrian Pfeiffer and Dr. Felix Köttig for fruitful discussions and help with the numerical methods. Furthermore, the authors would like to thank Dr. Steffen Hädrich, Dr. Cesar Jauregui-Misas, and Tobias Ulsperger for their help in the early stages of this work. This work was supported by the European Research Council (ERC) under the European Union's Horizon 2020 research and innovation programme (grant 835306, SALT), the Fraunhofer Cluster of Excellence Advanced Photon Sources (CAPS), the Helmholtz-Institute Jena, the U.S. Army Research Office (grant W911NF1910426), and the U.S. Air Force Office of Scientific Research (grant FA9550-15-10041).}

\section{Author details}

${ }^{1}$ Institute of Applied Physics, Abbe Center of Photonics, Friedrich-SchillerUniversität Jena, Albert-Einstein-Str. 15, 07745 Jena, Germany. ${ }^{2}$ HelmholtzInstitute Jena, Fröbelstieg 3, 07743 Jena, Germany. ${ }^{3} \mathrm{CREOL}$, College of Optics and Photonics, University of Central Florida, Orlando, FL 32816, USA.

${ }^{4}$ Fraunhofer Institute for Applied Optics and Precision Engineering, AlbertEinstein-Str. 7, 07745 Jena, Germany. ${ }^{5}$ Present address: Active Fiber Systems GmbH, Ernst-Ruska-Ring 17, 07745 Jena, Germany

\section{Author contributions}

J.L., J.R., M.G. and R.K. conceived and planned the experiment. The ultrafast fibre laser and the nonlinear pulse compression stage were built and optimised by M.G., T.H., M.L., Z.W. and C.G. The antiresonant hollow-core fibre was designed and drawn by J.A.-L., A.S. and R.A.-C. The HHG experiments were performed by M.G., T.H., R.K., C.L. and A.K. Simulations and data analysis were performed by M.G. with support from T.H. and R.K. All authors discussed and contributed to the interpretation of the results and to the writing of the manuscript. J.L. and J.R. supervised the project. J.L., R.A.-C. and J.R. acquired funding.

Conflict of interest

The authors declare that they have no conflict of interest.

Supplementary information The online version contains supplementary material available at https://doi.org/10.1038/s41377-021-00477-x.

Received: 21 September 2020 Revised: 7 January 2021 Accepted: 18 January 2021

Published online: 12 February 2021

\section{References}

1. Rundquist, A. et al. Phase-matched generation of coherent soft X-rays. Science 280, 1412-1415 (1998).

2. Young, L. et al. Roadmap of ultrafast x-ray atomic and molecular physics. J. Phys. B 51, 032003 (2018).

3. Pertot, $Y$. et al. Time-resolved X-ray absorption spectroscopy with a water window high-harmonic source. Science 355, 264-267 (2017).

4. Popmintchev, D. et al. Near- and extended-edge X-ray-absorption finestructure spectroscopy using ultrafast coherent high-order harmonic supercontinua. Phys. Rev. Lett. 120, 093002 (2018).

5. Rose, M. et al. Quantitative ptychographic bio-imaging in the water window. Opt. Express 26, 1237-1254 (2018).

6. Gaumnitz, T. et al. Streaking of 43-attosecond soft-X-ray pulses generated by a passively CEP-stable mid-infrared driver. Opt. Express 25, 27506-27518 (2017). 
7. Li, J. et al. 53-attosecond X-ray pulses reach the carbon K-edge. Nat. Commun. 8, 186 (2017).

8. Stein, G. J. et al. Water-window soft x-ray high-harmonic generation up to the nitrogen K-edge driven by a kHz, 2.1 um OPCPA source. J. Phys. B 49, 155601 (2016).

9. Feng, T. L. et al. $27 \mathrm{~W} 2.1 \mu \mathrm{m}$ OPCPA system for coherent soft $\mathrm{X}$-ray generation operating at $10 \mathrm{kHz}$. Opt. Express 28, 8724-8733 (2020).

10. Johnson, A. S. et al. High-flux soft x-ray harmonic generation from ionizationshaped few-cycle laser pulses. Sci. Adv. 4, eaar3761 (2018).

11. Cousin, S. L. et al. High-flux table-top soft x-ray source driven by sub-2-cycle, CEP stable, 1.85- $\mu \mathrm{m} 1-\mathrm{kHz}$ pulses for carbon K-edge spectroscopy. Opt. Lett. 39, 5383-5386 (2014).

12. Chen, M. C. et al. Bright, coherent, ultrafast soft X-ray harmonics spanning the water window from a tabletop light source. Phys. Rev. Lett. 105, 173901 (2010).

13. Shiner, A. D. Wavelength scaling of high harmonic generation efficiency. Phys. Rev. Lett. 103, 073902 (2009).

14. Hernández-García, C. et al. Group velocity matching in high-order harmonic generation driven by mid-infrared lasers. N. J. Phys. 18, 073031 (2016).

15. Pupeikis, J. et al. Water window soft $\mathrm{X}$-ray source enabled by a $25 \mathrm{~W}$ few-cycle $2.2 \mu \mathrm{m}$ OPCPA at $100 \mathrm{kHz}$. Optica 7, 168-171 (2020).

16. Rothhardt, J. et al. High-repetition-rate and high-photon-flux $70 \mathrm{eV}$ highharmonic source for coincidence ion imaging of gas-phase molecules. Opt. Express 24, 18133-18147 (2016)

17. Chiang, C. T. et al. Boosting laboratory photoelectron spectroscopy by megahertz high-order harmonics. N. J. Phys. 17, 013035 (2015).

18. Travers, J. C. et al. High-energy pulse self-compression and ultraviolet generation through soliton dynamics in hollow capillary fibres. Nat. Photonics $\mathbf{1 3}$, 547-554 (2019).

19. Schnürer, M. et al. Guiding and high-harmonic generation of sub-10-fs pulses in hollow-core fibers at $10^{15} \mathrm{~W} / \mathrm{cm}^{2}$. Appl. Phys. B 67, 263-266 (1998).

20. Travers, J. C. et al. Ultrafast nonlinear optics in gas-filled hollow-core photonic crystal fibers [Invited]. J. Optical Soc. Am. B 28, A11-A26 (2011).

21. Yu, F. \& Knight, J. C. Negative curvature hollow-core optical fiber. IEEE J. Sel. Top. Quantum Electron. 22, 4400610 (2016).

22. Wiegandt, F. et al. Quasi-phase-matched high-harmonic generation in gasfilled hollow-core photonic crystal fiber. Optica 6, 442-447 (2019).
23. Gebhardt, M. et al. Nonlinear pulse compression to 43 W GW-class few-cycle pulses at $2 \mu \mathrm{m}$ wavelength. Opt. Lett. 42, 4179-4182 (2017).

24. Saleh, M. F. et al. Theory of photoionization-induced blueshift of ultrashort solitons in gas-filled hollow-core photonic crystal fibers. Phys. Rev. Lett. 107, 203902 (2011).

25. Kazamias, S. et al. Pressure-induced phase matching in high-order harmonic generation. Phys. Rev. A 83, 063405 (2011).

26. Elu, U. et al. High average power and single-cycle pulses from a mid-IR optical parametric chirped pulse amplifier. Optica 4, 1024-1029 (2017).

27. Jin, C. et al. Waveforms for optimal sub-keV high-order harmonics with synthesized two- or three-colour laser fields. Nat. Commun. 5, 4003 (2014).

28. Michieletto, M. et al. Hollow-core fibers for high power pulse delivery. Opt. Express 24, 7103-7119 (2016).

29. Tani, F. et al. Effect of anti-crossings with cladding resonances on ultrafast nonlinear dynamics in gas-filled photonic crystal fibers. Photonics Res. 6, 84-88 (2018).

30. Livesey, R. G. Solution methods for gas flow in ducts through the whole pressure regime. Vacuum 76, 101-107 (2004).

31. Henningsen, J. \& Hald, J. Dynamics of gas flow in hollow core photonic bandgap fibers. Appl. Opt. 47, 2790-2797 (2008).

32. Tani, F., Travers, J. C. \& Russell, P. S. J. Multimode ultrafast nonlinear optics in optical waveguides: numerical modeling and experiments in kagomé photonic-crystal fiber. J. Optical Soc. Am. B 31, 311-320 (2014).

33. Ammosov, M. V., Delone, N. B. \& Kralnov, V. P. Tunnel ionization of complex atoms and of atomic ions in an alternating electromagnetic field. J. Exp. Theor. Phys. 64, 1191-1194 (1986). [Russian original - ZhETF, 91, 2008 (1986)].

34. Henke, B. L., Gullikson, E. M. \& Davis, J. C. X-ray interactions: photoabsorption, scattering, transmission, and reflection at $E=50-30,000 \mathrm{eV}, Z=1-92$. At. Data Nucl. Data Tables 54, 181-342 (1993).

35. Hädrich, S. et al. High photon flux table-top coherent extreme-ultraviolet source. Nat. Photonics 8, 779-783 (2014).

36. Frassetto, F. et al. Compact spectrometer for the analysis of high harmonics content of extreme-ultraviolet free-electron-laser radiation. Proc. SPIE 7802, Advances in X-Ray/EUV Optics and Components V, 780209 (SPIE, San Diego, CA, USA, 2010). 